\title{
Fabrication of long period fiber gratings of subnanometric bandwidth.
}

\author{
L. Poveda-Wong, J.L. Cruz*, M. Delgado-Pinar, X. Roselló-Mechó, A. \\ DÍEZ, M.V. ANDRÉS
}

Department of Applied Physics and Electromagnetism, University of Valencia, C/ Dr. Moliner 50, Burjassot 46100. Spain.

*Corresponding author: cruz@uv.es

Received XX Month XXXX; revised XX Month, XXXX; accepted XX Month XXXX; posted XX Month XXXX (Doc. ID XXXXX); published XX Month XXXX

\begin{abstract}
This paper reports on the fabrication of long period fiber gratings having subnanometric bandwidth in the 1500 nm spectral region. Large gratings have been photoinscribed in a high NA fiber, the grating pitch and the order of the HE cladding mode are optimized to produce gratings with a large number of periods and preventing the coupling to TE, TM or EH modes. Resonances with a FWHM of $0.83 \mathrm{~nm}$ and $0.68 \mathrm{~nm}$ have been achieved for gratings 15 and $20 \mathrm{~cm}$ long respectively, the free spectral range between transmission notches is $125 \mathrm{~nm}$. The polarization effects and the sensitivity of the gratings to temperature and to strain variations are presented as well. (C) 2015 Optical Society of America
\end{abstract}

OCIS codes: (060.2310) Fiber optics; (060.3735) Fiber Bragg gratings; (060.2370) Fiber optics sensors; (070.0070) Fourier optics and signal processing; (060.4230) Multiplexing.

http://dx.doi.org/

Gratings in optical fibers are periodic modulations of the fiber structure that couple energy between different modes. They are classified in two categories, Bragg gratings (BG), which have a periodicity of half a guided wavelength [1], and long period gratings (LPG) which have a period much longer than the wavelength [2]. The former couple energy at specific wavelengths between counter propagating modes (core-core, core - cladding, cladding-cladding) [3] and also to radiation modes [4], while the latter couple energy between co-propagating modes (core-core, core-cladding) $[5,6]$. The bandwidth of BGs ranges from few pm (for very large gratings) to hundreds of pm [7], however LPGs have much broader spectra because the number of periods of the gratings is much smaller; the bandwidth of LPGs in the S to L telecommunications bands ranges between several $\mathrm{nm}$ to a hundred $\mathrm{nm}[8,9,10]$. The reduction of the rejection bandwidth below $1 \mathrm{~nm}$ is usually achieved by association of gratings in coaxial interferometric configuration or in sampled configuration [11, 12], however the individual gratings have a large bandwidth and the spectral sub-bands cannot be designed or handled independently.
There is still a need of fabricating long period fiber gratings with narrow rejection bands for core-cladding conversion, these gratings would find applications in signal processing [9, 13], DWDM components and rejection filters for telecommunications $[14,15]$ or multiplexing series of sensors [16]. This paper presents what is, up to our knowledge, the first demonstration of a long period grating written in an optical fiber with a $3 \mathrm{~dB}$ bandwidth below the frontier of $1 \mathrm{~nm}$ at $1527 \mathrm{~nm}$ and more than $100 \mathrm{~nm}$ of free spectral range. The paper discusses first the fabrication procedure, then the grating characteristics and finally the sensitivity to thermal and mechanical perturbations.

The $3 \mathrm{~dB}$ bandwidth of a narrowband LPG can be expressed in following forms:

$$
\frac{\Delta \lambda_{3 d B}}{\lambda_{\text {res }}} \approx 0.8 \frac{\lambda_{\text {res }}}{\left(n_{g c}-n_{g l}\right) L} \approx \frac{0.8 \Lambda}{L}=\frac{0.8}{N}
$$

Where $\lambda_{\text {res }}$ is the coupling wavelength, $\Lambda$ is the grating period, $n_{g c}$ and $n_{g l}$ are the group indices of the core mode and the $l$-th cladding mode, $L$ is the grating length and $N$ is the number of periods. Since $n_{g}=n_{\text {eff }}-\lambda \partial n_{\text {eff }} / \partial \lambda$, being $n_{\text {eff }}$ the mode effective index, one can assume that $n_{g} \cong n_{\text {eff }}$ in most cases. The key for bandwidth reduction is the grating length, but fabrication of long gratings requires high precision translation stages, good laser stability and perfect fiber uniformity. The bandwidth scales with the operation wavelength, making more challenging the $1500 \mathrm{~nm}$ spectral region than shorter wavelength ranges. The bandwidth decreases as well with the difference of modal indices, fibers of high NA have a higher index core that results in gratings of smaller bandwidth for a given mode order. The order of the cladding mode determines the grating bandwidth as well (the index difference rises with the mode order), but the grating mode is often chosen with other criteria: the dispersion turning point of resonances, the coupling strength or the free spectral range between resonances. The dispersion at the turning point affects the bandwidth as the dispersive term included in the group index of eq. 1 states. Observe that the bandwidth can be reduced as well in conventional (low NA) fibers by using periods of few tens of $\mu \mathrm{m}$ and coupling to very high order modes [17], but very low periods reduce the distance between adjacent resonances (which is given approximately by 
$\left.\lambda_{\text {res },-}-\lambda_{\text {res, },+1} \cong\left(n_{\text {eff }, l+1}-n_{\text {eff, }, l}\right) \Lambda\right)$ despite the higher index difference between modes. Furthermore very large order modes compete with unwanted modes (modes other than the HE1m) which have coupling factors comparable to adjacent $\mathrm{HE}_{1 \mathrm{~m}}$ modes. Our approach, as it is demonstrated in the paper, overcomes those problems.

A fiber with NA=0.29 supplied by Fibercore was used in this experiment (product number SM1500-4.2/125). The fiber had a cutoff wavelength of $1387 \mathrm{~nm}$, a modal field diameter of $4.2 \mu \mathrm{m}$ and an attenuation of $1.95 \mathrm{~dB} / \mathrm{Km}$ at $1550 \mathrm{~nm}$. A Bragg grating was written to get information about the effective indices of the cladding modes. The BG was inscribed scanning a UV beam over the fiber through a phase mask. A CW laser at $244 \mathrm{~nm}$ was used with a power of $83 \mathrm{~mW}$, the beam was focused by a cylindrical lens and the flux of energy in the fiber core was estimated to be 160 $\mathrm{J} / \mathrm{mm}^{2}$. The grating spectrum is shown in Fig.1. The core mode $\mathrm{HE}_{11}$ is reflected at $1556.9 \mathrm{~nm}$ and its resonance depth exceeds -38 $\mathrm{dB}$. Several couplings to cladding modes appear at shorter wavelengths, the main series of resonances have a depth of $-1.7 \mathrm{~dB}$ around $1540 \mathrm{~nm}$, this series corresponds to cladding modes $\mathrm{HE}_{1 \mathrm{~m}}$ and $\mathrm{EH}_{1 \mathrm{~m}}$ [18]. A secondary series of notches of about $-0.1 \mathrm{~dB}$ deep appears between the notches of the main series, this series corresponds to modes $\mathrm{TE}_{0 \mathrm{~m}}, \mathrm{TM}_{0 \mathrm{~m}}$ and $\mathrm{HE}_{2 \mathrm{~m}}[19,20]$, these resonances usually appear in strong gratings because of the fiber imperfections or fabrication inaccuracies (i.e. core eccentricity or beam misalignment).
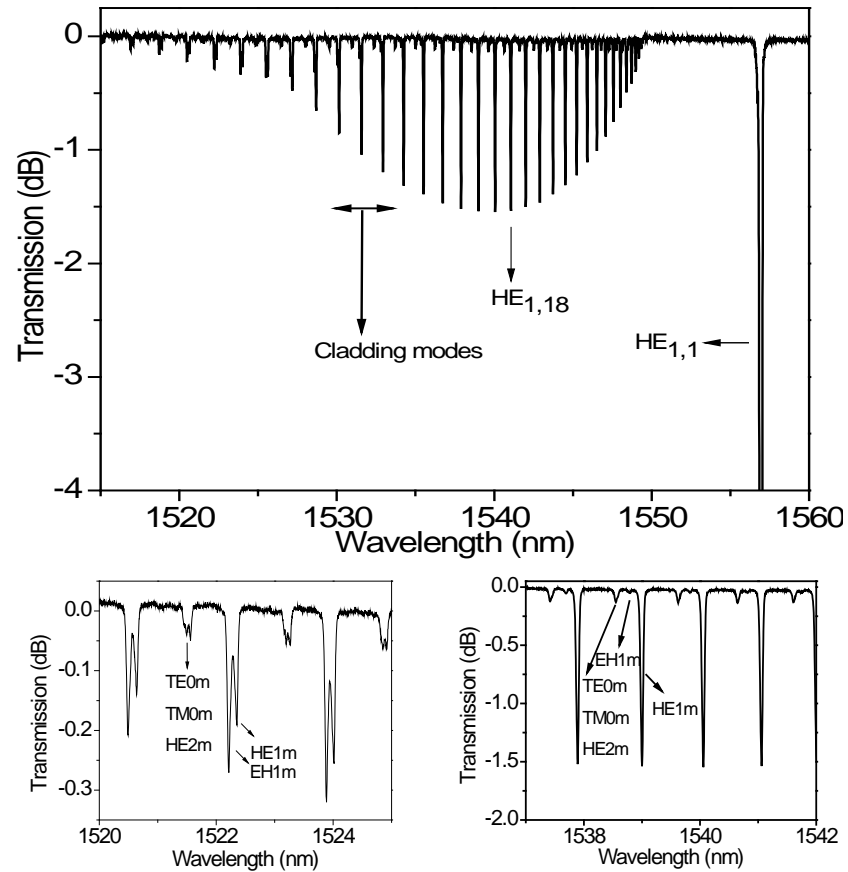

Fig. 1. Bragg grating spectrum, the vertical scale is magnified to highlight cladding modes resonances. Full wavelength range (top), detail at $1522 \mathrm{~nm}$ (bottom left) and detail at $1540 \mathrm{~nm}$ (bottom right).

The coupling to $\mathrm{HE}_{1 \mathrm{~m}}$ and $\mathrm{EH}_{1 \mathrm{~m}}$ modes can be resolved in the first series of resonances as it is shown in the bottom details of Fig. 1. Around $1522 \mathrm{~nm}$ the coupling to $\mathrm{EH}_{1 \mathrm{~m}}$ modes is stronger than to $\mathrm{HE}_{1 \mathrm{~m}}$ modes, however the $\mathrm{EH}_{1 \mathrm{~m}}$ modes reduce their coupling strength to near zero around $1540 \mathrm{~nm}$ while the $\mathrm{HE}_{1 \mathrm{~m}}$ modes reach maximum coupling (see the bottom right detail of Fig. 1). No splitting is observed for the $\mathrm{HE}_{1 \mathrm{~m}}$ resonances around $1540 \mathrm{~nm}$, which indicates that other modes $\left(\mathrm{EH}_{3 \mathrm{~m}}, \mathrm{HE}_{3 \mathrm{~m}}\right)$ are either quasidegenerated or weakly coupled. The $\mathrm{HE}_{1 \mathrm{~m}}$ modes experience a polarization dependent shift of $4 \mathrm{pm}$ in the $1540 \mathrm{~nm}$ region (not shown in graphs) that can be due to the birefringence induced by UV radiation or to the residual birefringence of the fiber.

The effective indices of the core and cladding modes can be calculated from the resonant wavelengths of the BG assuming that the fundamental core mode has little variation in the wavelength range of the cladding modes. Although this is an approximation, it gives sufficient precision for the lowest order modes, allowing for the design of LPGs whose resonance has to lie in the spectral region of the $\mathrm{BG}$ resonances (this will be verified later). The refractive indices and the resonant wavelengths of $\mathrm{HE}_{1 \mathrm{~m}}$ modes are presented in Fig. 2. The index difference between the core and cladding modes is large for this particular fiber; therefore, the period of the LPGs will be short: tens of microns to couple in S or C bands.

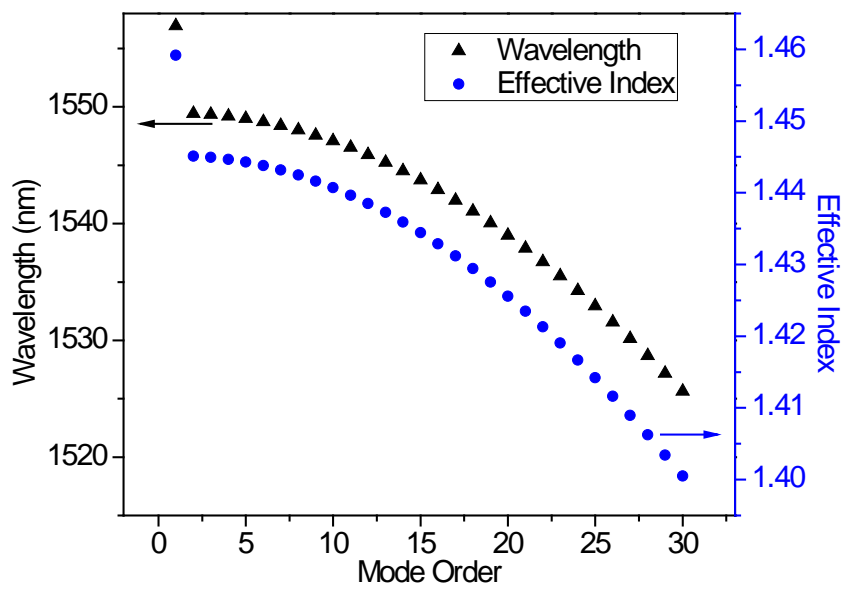

Fig. 2. Resonant wavelengths and refractive indices of $\mathrm{HE}_{1 \mathrm{~m}}$ modes.

The effective indices of the modes were numerically calculated by solving the Maxwell equations in a realistic fiber. This calculation allows us to make sure that the cladding resonances of lowest order $\left(\mathrm{HE}_{1,2}\right.$ and $\left.\mathrm{HE}_{1,3}\right)$ have been correctly identified in Fig. 1. The results of the simulation are in good agreement with experimental data as it is shown in Fig. 3.

The maximum coupling strength corresponds to $\mathrm{HE}_{1 \mathrm{~m}}$ modes between $m=15$ and $m=20$ as can be observed in Fig. 1 . On the contrary, the relative strength of their neighboring $\mathrm{EH}_{1 \mathrm{~m}}$ modes is much smaller; therefore, LPGs based on this set of modes $\left(\mathrm{HE}_{1,15}\right.$ to $\mathrm{HE}_{1,20}$ ) will have a large free spectral range between resonances and will be less likely to present unwanted resonances caused by $\mathrm{EH}, \mathrm{TE}$ or TM modes that might appear due to fabrication inaccuracies. Additionally, the $\lambda_{\text {res versus }} \Lambda$ diagram of Fig. 3 does not show the dispersion turning point for this set of modes, therefore the resonances of the LPG will not experience the ultrawide spectral broadening that other modes of higher order could have. The free spectral range between consecutive resonances expected from Fig. 3 is larger than $100 \mathrm{~nm}$. 

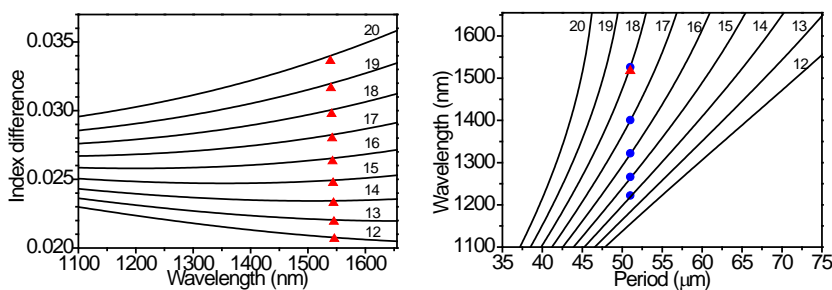

Fig. 3. Left: effective index difference between the $\mathrm{HE}_{11}$ mode and $\mathrm{HE}_{1 \mathrm{~m}}$ modes for $m=12$ to 20 (lines: theory, dots: experimental data from $B G$ ). Right: Resonant wavelength as function of the LPG period for $\mathrm{HE}_{11}$ to $\mathrm{HE}_{1 \mathrm{~m}}$ couplings for $\mathrm{m}=12$ to 20 (lines: theory, triangle: expected form BG, circles: experimental data from LPG).

The LPGs of this experiment were designed to couple the mode $\mathrm{HE}_{1,18}$; thus, the grating period was set at $\Lambda=51 \mu \mathrm{m}$. Taking into account the indices of Fig. 2 , the $\mathrm{HE}_{1,18}$ mode was expected to have a resonance at $1517 \mathrm{~nm}$. Mode 18 was chosen as a balance of large intermodal distance, short period (high $\mathrm{N}$ ) and moderate slope of the $\lambda_{\text {res }}$ versus period curve; mode 20 with shorter period would have larger free spectral range according to Fig. 3 but it might also have larger bandwidth because it is closer to the dispersion turning point (where $\partial \lambda_{\text {res }} / \partial \Lambda=\infty$ and $\Delta \mathrm{n}_{\mathrm{g}}=\mathrm{n}_{\mathrm{gc}}-\mathrm{n}_{\mathrm{gl}}=0$ ). The grating was made point by point, by irradiating the fiber through a slit 25 $\mu \mathrm{m}$ wide. The slit diffracts the UV beam with a far-field divergence of $19.5 \mathrm{mrad}$, the fiber core lies in Fresnel's diffraction zone. The UV beam was again focused along the fiber axis with a cylindrical lens and its power was $57 \mathrm{~mW}$; half a period of the grating was irradiated for a lapse of time of $1000 \mathrm{~ms}$ with a flux of energy of 1.5 $\mathrm{J} / \mathrm{mm}^{2}$, the second half of the period was quickly scanned in about $10 \mathrm{~ms}$. The scan process was controlled by an encoded motor from Physik Instrumente (product M525.22).

The grating growth was monitored during fabrication: the transmission notch as a function of the grating length is shown in Fig. 4. A super-luminescent diode partially polarized was used as light source, light was polarized by an external polarizer with an extinction ratio of $35 \mathrm{~dB}$ at $1550 \mathrm{~nm}$. The polarization state had to be carefully adjusted during the grating inscription: the spectral notch is broad when the grating is short and the polarization dependency is undistinguishable; however, when the notch depth exceeds $3 \mathrm{~dB}$, two close transmission notches that correspond to the slow and fast axes of the fiber can be distinguished and the polarization has to be matched to one of them in order to adjust the grating length for optimum coupling. The resulting grating was $150 \mathrm{~mm}$ in length.

The grating presents two transmission notches that correspond to the slow and fast axis of the fiber, each resonance has a $3 \mathrm{~dB}$ bandwidth of $0.83 \mathrm{~nm}$. The upper band is centered at $1526.78 \mathrm{~nm}$ and has a depth of $-30.2 \mathrm{~dB}$, the lower band is shifted $0.97 \mathrm{~nm}$ and has a depth of $-30.9 \mathrm{~dB}$. The spectra were measured with a resolution of $18 \mathrm{pm}$ and the bottom of the notches is well resolved. The insertion loss of the grating measured out of the band is less than $0.1 \mathrm{~dB}$. The suppressed bands have sidelobes $-1.2 \mathrm{~dB}$ deep, their asymmetry indicates an uneven chirp in the grating. The bandwidth expected from equation 1 is $0.42 \mathrm{~nm}$, the discrepancy is discussed later. The resonant wavelengths of the LPG are $0.7 \%$ higher than expected from the refractive indices of the BG in Fig.2, notice that the LPG was made with lower UV energy and it has smaller average index than the $\mathrm{BG}$, hence, shorter resonant wavelength.
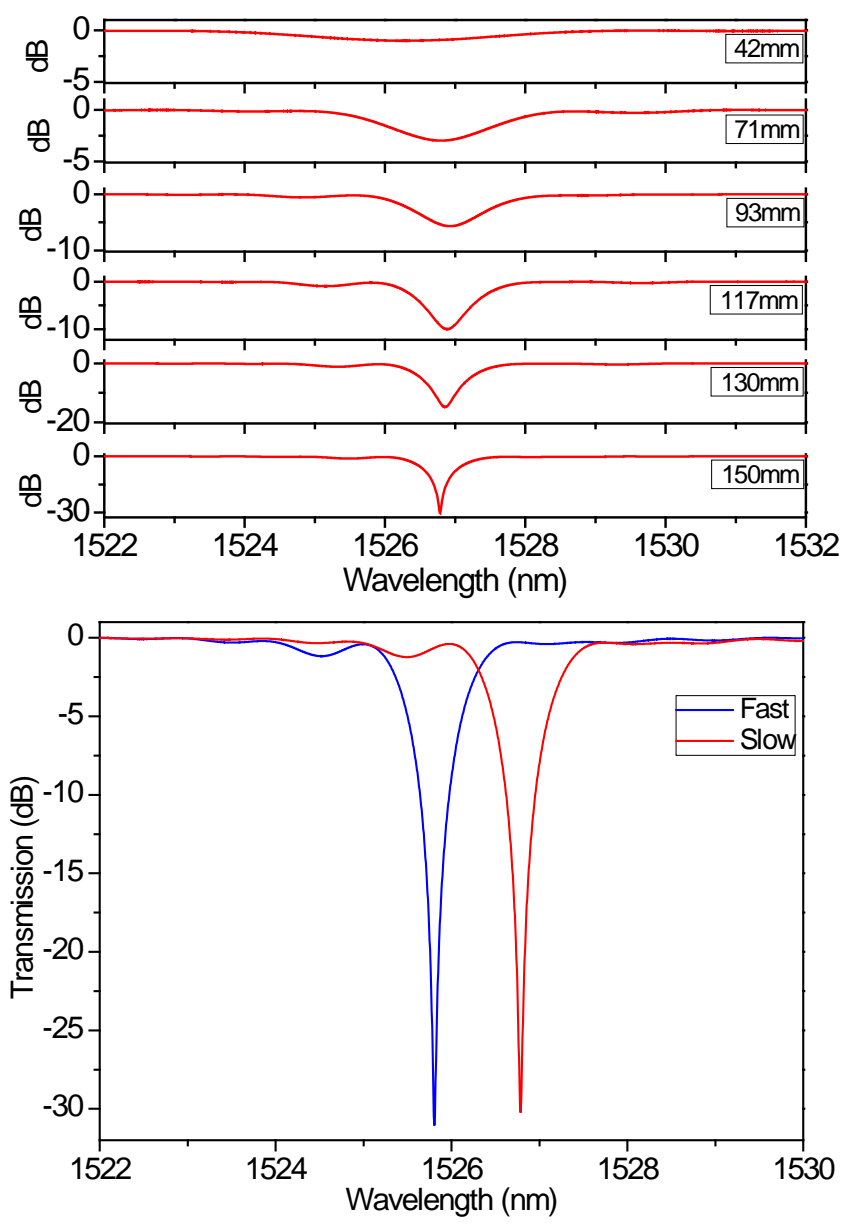

Fig. 4. LPG spectrum as function of the grating length (top). Polarization dependency for $150 \mathrm{~mm}$ length (bottom).

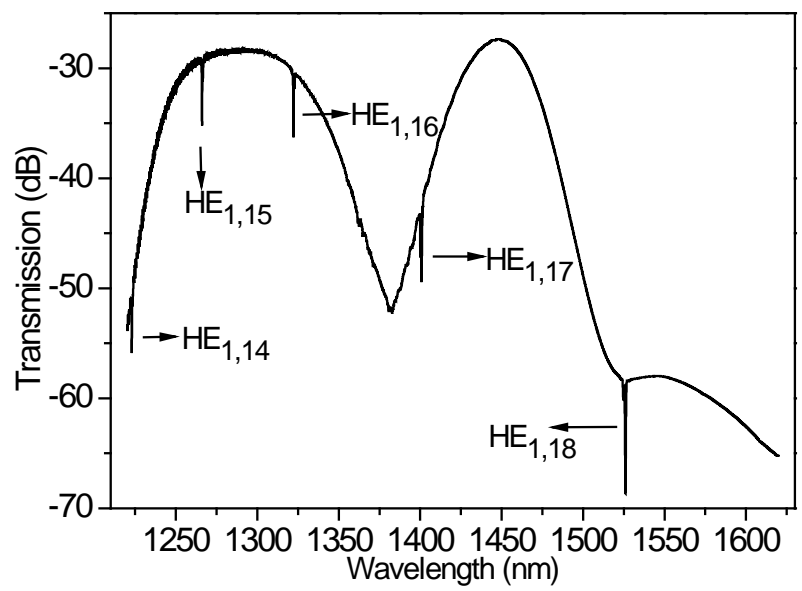

Fig. 5. Spectrum showing resonances of different cladding modes.

After fabrication, the LPGs spectrum was measured in a span of $400 \mathrm{~nm}$ to identify couplings to other modes, see Fig. 5 . The closest resonance appears at $1400.7 \mathrm{~nm}$ and corresponds to mode $\mathrm{HE}_{1,17}$ in good agreement with predictions from Fig. 3. No sign of coupling to $\mathrm{EH}_{1 \mathrm{~m}}, \mathrm{HE}_{2 \mathrm{~m}}, \mathrm{TM}_{0 \mathrm{~m}}$ or $\mathrm{TE}_{0 \mathrm{~m}}$ was observed between consecutive 
HE resonances; consequently the LPG has a free spectral range of $125 \mathrm{~nm}$ available for wavelength multiplexing. The bottom of the resonances in Fig. 5 is not well resolved because the set of diodes used as light source was partially polarized.

We have also written strong gratings impinging the UV beam at a small angle with respect to the fiber axis, the resulting asymmetric LPGs present an additional resonance located $1 \mathrm{~nm}$ below the fundamental $\mathrm{HE}_{1,18}$ resonance; we believe this closest resonance corresponds to a mode $\mathrm{HE}_{3 \mathrm{~m}}$ which is almost degenerated with the $\mathrm{HE}_{1,18}[19,20]$. It was as well observed in gratings fabricated with different UV powers that the spectral separation between polarization bands remains unalterable, this indicates that polarization splitting is due to the intrinsic birefringence of the fiber rather than induced by the UV radiation (at least for the energy levels of our experiments).

The sensitivity of the LPG to temperature and axial strain was measured after annealing the grating at $90{ }^{\circ} \mathrm{C}$ for $24 \mathrm{~h}$ in order to stabilize the refractive index profile photoinduced in the fiber during the grating fabrication. As it is shown in Fig. 6 (left), the sensitivities of this resonance are $91 \mathrm{pm} /{ }^{\circ} \mathrm{C}$ and $1.0 \mathrm{pm} / \mu \varepsilon$.

As it has been commented before, the LPGs have an unwanted chirp due to fabrication inaccuracies. We have identified three causes of error: (1) the stability of the room temperature, (2) the fluctuations of the UV power (laser power or the spatial beam stability) and (3) the axial uniformity of the pristine fiber. A temperature variation of $\pm 1^{\circ} \mathrm{C}$ results in a variation of the resonant wavelength of $\pm 90 \mathrm{pm}$. The refractive index modulation of the grating was estimated to be $2.3 \times 10^{-5}$; assuming a linear dependence on the UV power, a variation of $\pm 2 \mathrm{~mW}$ results in a chirp of $\pm 40 \mathrm{pm}$. Furthermore, short pieces of fiber have a random variation of the core radius and refractive indices [21]; these irregularities could change the difference between effective indices up to $10^{-6}$, and this random variation results in a chirp of $\pm 50 \mathrm{pm}$. The overall effect is an rms error of $\pm 110 \mathrm{pm}$ along the grating.

Because of this random chirp, the quality of the gratings worsens as the bandwidth is reduced. As an example, we show in Fig. 6 (right) the spectrum of a $200 \mathrm{~mm}$ long grating. The spectral bandwidth is $0.68 \mathrm{~nm}$ and $0.70 \mathrm{~nm}$ for the fast and slow axis; however, the sidelobes are $-1.8 \mathrm{~dB}$ and $-1.9 \mathrm{~dB}$ deep and the resonances have $-18.0 \mathrm{~dB}$ and $-16.7 \mathrm{~dB}$ for the two polarizations. Attempts to increase the coupling of this grating were made, but we found systematically that sidelobes grew further and invaded the main resonance broadening the spectrum.
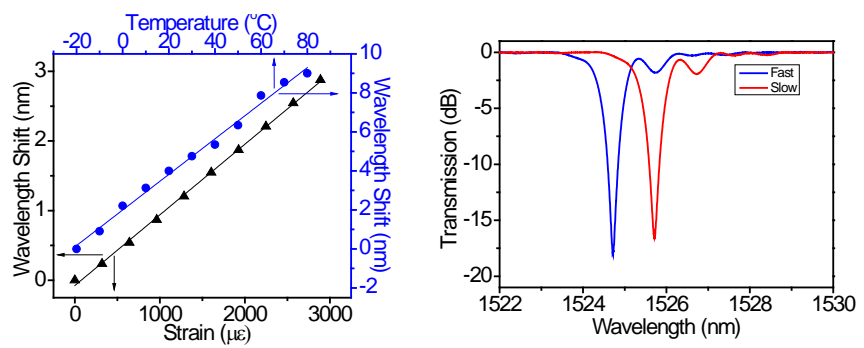

Fig. 6. Left: LPG response to temperature and strain (lines: linear fit). Right: Spectrum of a $200 \mathrm{~mm}$ length LPG. The 3dB bandwidths are $0.68 \mathrm{~nm}$ and $0.70 \mathrm{~nm}$ for the fast and slow axis.
In summary, we have demonstrated narrowband LPGs fabricated using a high NA fiber whose core and cladding modes have differences between effective indices larger than those of low NA fibers. The period of the gratings is short in this kind of fiber, hence the number of periods for given length is higher; and, as a result, the bandwidth is reduced. Bandwidths of $0.83 \mathrm{~nm}$ have been achieved in $15 \mathrm{~cm}$ long gratings which have excellent spectral quality; the bandwidth has been reduced to $0.68 \mathrm{~nm}$ in gratings 20 $\mathrm{cm}$ long, but the spectral quality is slightly poorer. The spectrum has a free spectral range of $125 \mathrm{~nm}$ and the suppressed band is polarization split into two bands $0.97 \mathrm{~nm}$ apart. The resonance corresponding to the $\mathrm{HE}_{1,18}$ mode has sensitivities to temperature and strain of $91 \mathrm{pm} /{ }^{\circ} \mathrm{C}$ and $1.0 \mathrm{pm} / \mu \varepsilon$. The main limitations for further improvement are the stability of the UV beam and the room temperature during fabrication, as well as the quality of the pristine fiber.

Funding. Ministerio de Economia y Competitividad of Spain and FEDER funds (project TEC2013-46643-C2-1-R), and the Generalitat Valenciana (project PROMETEOII /2014/072).

\section{References}

1. K. O.Hill, Y. Fujii, D. C. Johnson, and B. S. Kawasaki, Appl. Phys. Lett. 32, 647 (1978).

2. J. N. Blake, B. Y. Kim, and H. J. Shaw, Opt. Lett. 11, 177 (1986).

3. D. Sáez-Rodriguez, J. L. Cruz, A. Díez, and M. V. Andrés, Opt. Lett. 36, 1518 (2011).

4. T. Erdogan and J. E. Sipe, J. Opt. Soc. Am. A, 13, 396 (1996).

5. K. O.Hill, B. Malo, K. A. Vinerberg, F. Bolideau, D.C. Jhonson, and I. Skinner, Electron. Lett. 26, 1270 (1990).

6. A. M. Vengsarkar,P.J. Lemaire, J. B. Judkins, V. Bhatia, T. Erdogan, and J. E. Sipe, J. Lightwave Thecnol. 14, 58 (1996).

7. M. Gagné, S. Loranger, J. Lapointe, and R. Kashyap, Opt. Express, 22, 387 (2014).

8. R. Kashyap, A. Swanton, and R. P. Smith, Electron. Lett. 35, 1871 (1999).

9. R. Slavik, Y. Park, M. Kulishov, R. Morandotti, and J. Azaña, Opt. Express, 14, 10699 (2006).

10. S. Ramachandran, S. Ghalmi, Z. Wang, and M. Yan, Opt. Lett. 27, 1678 (2002).

11. Y. Liu, J.A.R. Williams, L. Zhang, and I. Bennion, Opt. Communications 164, 27 (1999).

12. X. Dong, N. Pan, P. Shum, and C.C. Chan, Microwave Opt. Thecnol. Lett., 51, 2401 (2009).

13. L. Poveda-Wong, A. Carrascosa, C. Cuadrado-Laborde, J. L. Cruz, A. Díez, and M. V.Andrés, Opt. Communications 371, 89, (2016).

14. X. Dong, X. Yang, and P. Shum, IEEE Photonics Tehcnol. Lett. 17, 795, (2005).

15. Y. J. He, Y. L. Lo, and J. F. Huang, Opt. Engineering 45, 125001, (2006).

16. T. Allsop, K. Carroll, G. Lloyd, D.J. Webb, M. Miller, and I. Bennion, J. Biomedical Opt. 12, 064003 (2007).

17. Z. Zhang, C. Mou, Z. Yan, Y. Wang, K. Zhou, and Z. Zhang, Opt. Express 26, 1353, (2015).

18. G. Yin, S. Lou, Q. Li, and H. Zou, Opt. Laser Technol. 48, 60 (2013).

19. J. Thomas, N. Jovanovec, R. G. Becker, G.D., Marshal, M. J. Withford, A. Tünnermann, S. Nolte, and M. J. Steel, Opt. Express 19, 325 (2011).

20. J. Thomas, N. Jovanovec, R. G. Becker, G.D., Marshal, M. J. Withford, A. Tünnermann, S. Nolte, and M. J. Steel, Opt. Express 20, 21434 (2012).

21. E. P. Alcusa-Sáez, A. Díez, M. González-Herráez, and M.V. Andrés, Opt. Express 23, 7345 (2015). 


\section{References}

1. K. O.Hill, Y. Fujii, D. C. Johnson, and B. S. Kawasaki, "Photosensitivity in optical fiber waveguides: application to reflection fiber fabrication", Appl. Phys. Lett. 32, 647 (1978).

2. J. N. Blake, B. Y. Kim, and H. J. Shaw, "Fiber-optic modal coupler using periodic microbending", Opt. Lett. 11, 177 (1986).

3. D. Sáez-Rodriguez, J. L. Cruz, A. Díez, and M. V. Andrés, “Coupling between counterpropagating cladding modes in fiber Bragg gratings", Opt. Lett. 36, 1518 (2011)

4. T. Erdogan and J. E. Sipe, "Tilted fiber phase gratings", J. Opt. Soc. Am. A, 13, 396 (1996).

5. K. O.Hill, B. Malo, K. A. Vinerberg, F. Bolideau, D.C. Jhonson, and I. Skinner, "Efficient mode conversion in telecommunication fiber using externally written gratings", Electron. Lett. 26, 1270 (1990).

6. A. M. Vengsarkar,P.J. Lemaire, J. B. Judkins, V. Bhatia, T. Erdogan, and J. E. Sipe, "Long period fiber gratotings as band rejection filters", J. Lightwave Thecnol. 14, 58 (1996).

7. M. Gagné, S. Loranger, J. Lapointe, and R. Kashyap, "Fabrication of high quality, ultra-long fiber Bragg gratings: up to 2 million periods in phase ", Opt. Express, 22, 387 (2014).

8. R. Kashyap, A. Swanton, and R. P. Smith, "Infinity length fiber gratings Electron. Lett. 35, 1871 (1999).

9. R. Slavik, Y. Park, M. Kulishov, R. Morandotti, and J. Azaña, "Ultrafast alloptical differenciators", Opt. Express, 14, 10699 (2006).

10. S. Ramachandran, S. Ghalmi, Z. Wang, and M. Yan "Band-selection filters with concatenated long-period gratings in few-mode fibers", Opt. Lett. 27, 1678 (2002).

11. Y. Liu, J.A.R. Williams, L. Zhang, and I. Bennion, "Phase shifted and cascaded long-period fiber gratings", Opt. Communications 164, 27 (1999).

12. X. Dong, N. Pan, P. Shum, and C.C. Chan, "Sampled Lond-period grating filters with narrow stop bands", Microwave Opt. Thecnol. Lett., 51, 2401 (2009).

13. L. Poveda-Wong, A. Carrascosa, C. Cuadrado-Laborde, J. L. Cruz, A. Díez, and M. V.Andrés, "Instantaneous Frequency Measurement by in-fiber 0.5th Order Fractional Differentiation", Opt. Communications 371, 89, (2016).

14. X. Dong, X. Yang, and P. Shum "Tunable WDM filter with 0.8-nm channel spacing using a pair of long-period fiber gratings" IEEE Photonics Tehcnol. Lett. 17, 795, (2005).

15. Y. J. He, Y. L. Lo, and J. F. Huang, "Bandwidth analysis of long-period fiber gratings for high-order cladding mode and its application to an optical add-drop multiplexer", Opt. Engineering 45, 125001, (2006).

16. T. Allsop, K. Carroll, G. Lloyd, D.J. Webb, M. Miller, and I. Bennion, "Application of long-period-grating sensors to respiratory plethysmography" J. Biomedical Opt. 12, 064003 (2007).

17. Z. Zhang, C. Mou, Z. Yan, Y. Wang, K. Zhou, and Z. Zhang, "Switchable dual-wavelength $Q$-switched and mode-locked fiber lasers using a largeangle tilted fiber grating", Opt. Express 26, 1353, (2015).

18. G. Yin, S. Lou, Q. Li, and H. Zou, "Therory analisys of mode coupling in tilted long period fiber grating based full vector coupled mode theory", Opt. Laser Technol. 48, 60 (2013).

19. J. Thomas, N. Jovanovec, R. G. Becker, G.D., Marshal, M. J. Withford, A. Tünnermann, S. Nolte, and M. J. Steel, “Cladding mode coupling in highly localize Bragg gratings: modal properties and transmission spectra", Opt. Express 19, 325 (2011).

20. J. Thomas, N. Jovanovec, R. G. Becker, G.D., Marshal, M. J. Withford, A. Tünnermann, S. Nolte, and M. J. Steel, "Cladding mode coupling in highly localize Bragg gratings II: complete victoria analysis", Opt. Express 20, 21434 (2012).

21. E. P. Alcusa-Sáez, A. Díez, M. González-Herráez, and M.V. Andrés, "Improoved time-resolved acousto-optic technique for optical fiber analysis of axial non-uniformities by using edge interrogation", Opt. Express 23, 7345 (2015). 